Author has nothing to disclose with regard to commercial support.
Bo Yang, MD, PhD

Department of Cardiac Surgery

Michigan Medicine

Ann Arbor, Mich

\title{
References
}

1. Motekallemi A, Dell'Aquila AM, Rukosujew A. The modified adventitial inversion technique as an alternative method for aortic repair in acute type A aortic dissection: balancing complexity and effectiveness. J Thorac Cardiovasc Surg. 2020;159:e103.

2. Tanaka K, Morioka K, Li W, Yamada N, Takamori A, Handa M, et al. Adventitial inversion technique without the aid of biologic glue or Teflon buttress for acute type A aortic dissection. Eur J Cardiothorac Surg. 2005; 28:864-9.

3. Yang B. Is biologic glue the inexperienced surgeon's best friend? J Thorac Cardiovasc Surg. 2019;157:e122-4.

4. Yang B, Malik A, Waidley V, Kleeman KC, Wu X, Norton EL, et al. Short-term outcomes of a simple and effective approach to aortic root and arch repair in acute type A aortic dissection. J Thorac Cardiovasc Surg. 2018;155:1360-13670.e1.

5. Mackman N, Tilley RE, Key NS. Role of the extrinsic pathway of blood coagulation in hemostasis and thrombosis. Arterioscler Thromb Vasc Biol. 2007; 27:1687-93.

https://doi.org/10.1016/j.jtcvs.2019.07.034

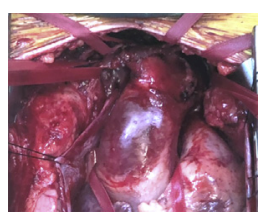

\section{DISSECTION FOR DISSECTION: LIMITED FOR DELIBERATE CABROL FISTULA OR EXTENDED FOR OPTIMAL EXPOSURE?}

\section{To the Editor:}

In the current issue of the Journal, Zhang and colleagues ${ }^{1}$ reported their experience in applying the modified Cabrol fistula in surgical repair of acute type A aortic dissection (aTAAD), and the authors should be commended for their good management of this high-risk surgical emergency, especially for their $0 \%$ reexploration to check bleeding and low 30-day mortality (2.6\%) in the fistula group. ${ }^{1}$ Although not emphasized in this article, several intraoperative characteristics were also impressive, such as many complex aortic reconstructions involving the root or arch performed and their short aortic crossclamp and selective cerebral perfusion times.

Although indications of the modified Cabrol fistula were mentioned in the study, the decision was made before aortic reconstruction, irrespective of inaccessible surgical bleeding after the procedure. Thus, most fistulas should be considered "deliberate." Besides proper closure of the transverse pericardial sinus (although difficult, it could be

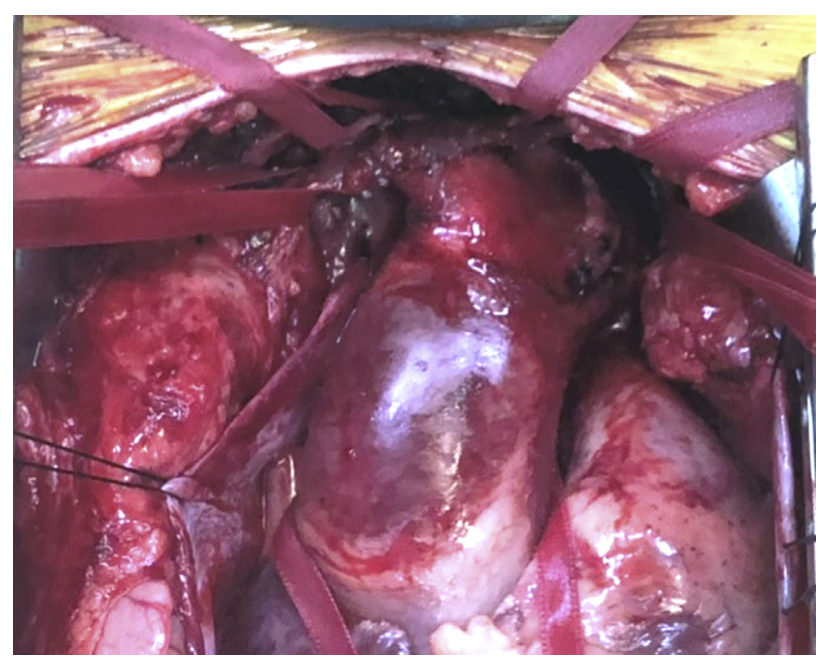

FIGURE 1. Optimal surgical exposure during repair of aTAAD is accomplished by removal of the entire thymic remnant, wide opening of the cranial portion of the pericardial reflection, and extended dissection of the anterior mediastinal tissue. Along with the ascending aorta, the structures exposed and looped by the tapes are (from left to right) the innominate vein, innominate artery, left common carotid artery, left subclavian artery, and vagus nerve.

performed after aortic graft implantation), securely anchoring a pericardial patch to cardiac or mediastinal structures is the decisive factor to successfully restricts exsanguination to the factitious space, followed by creating a draining fistula connected to the venous system. A limited mediastinal dissection might be necessary for "deliberate" Cabrol fistulas using the "anterior pericardial recess coverage" technique. It would especially be true in patients with aortic reconstruction extending to the arch, as shown in Figure 2, $C$ in the article, in whom the innominate vein has to be sutured to the cranial part of the mediastinal tissue after being anchored by the pericardial patch caudally, which might be impossible once extended dissection has been performed for better exposure of the arch branches and distal aortic segments.

Nevertheless, to avoid bleeding complications after surgical repair of aTAAD, the fundamental remains to prevent it in advance and to fix it immaculately, both of which could be carried out with good exposure (Figure 1). Good exposure not only makes suturing the fragile aortic tissue more precise but also facilitates the subsequent hemostatic process. Repairing any bleeders after aortic reconstruction for aTAAD in a poorly exposed surgical field could be not only difficult but also dangerous, because excessive traction of poorly mobile aorta might result in catastrophic breakdown at the anastomosis sites. We recommend the modified Cabrol fistula as a salvage procedure for patients with inaccessible surgical bleeding after surgical repair of aTAAD, but not being unduly applied 
Authors have nothing to disclose with regard to commercial support.

to all medical oozing caused by prolonged cardiopulmonary bypass, systemic deep hypothermia, or aortic dissection associated consumption coagulopathy.

Despite improved surgical outcomes of aTAAD and shift of focus to managing malperfusion and late aortic remodeling nowadays, saving the patient's life remains the primary goal in managing this potentially fatal disease. Depending on the surgeon's experiences, every effort to eliminate bleeding complications after surgical repair of aTAAD should be encouraged, including the Cabrol fistula and its modifications and alternatives. ${ }^{2-5}$ Although there were no short-term complications associated with the modified Cabrol fistula in this study, reports on the long-term adverse events, if any, would be informative, especially infections due to foreign materials and compressive effects of the residual hematoma.

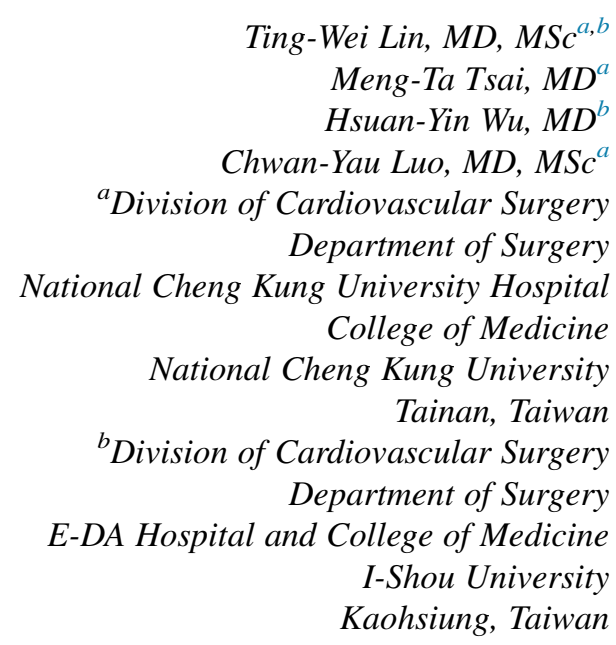

\section{References}

1. Zhang H, Wu X, Fang G, Qiu Z, Chen LW. Is it justified to apply a modified Cabrol fistula in surgical repair of acute type A aortic dissection? J Thorac Cardiovasc Surg. 2019;158:792-802.

2. Lin TW, Tsai MT, Hu YN, Lin WH, Wang WM, Luo CY, et al. Postoperative extracorporeal membrane oxygenation support for acute type A aortic dissection. Ann Thorac Surg. 2017; 104:827-33

3. Copeland JG III, Rosado LJ, Snyder SL. New technique for improving hemostasis in aortic root replacement with composite graft. Ann Thorac Surg. 1993;55:1027-9.

4. Posacioglu H, Apaydin AZ, Calkavur T, Yagdi T, Islamoglu F. Perigraft to right atrial shunt by using autologous pericardium for control of bleeding in acute type A dissections. Ann Thorac Surg. 2002;74:1071-4.

5. Lin TW, Tsai MT, Wu HY. "Mantle-style" modification of Cabrol shunt for hemostasis after extended aortic reconstruction in acute type A aortic dissection. Gen Thorac Cardiovasc Surg. 2019;67:1001-5.

https://doi.org/10.1016/j.jtcvs.2019.07.056
Author has nothing to disclose with regard to commercial support.

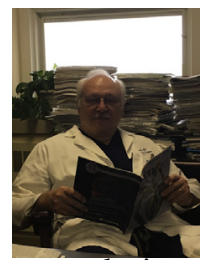

\section{REPLY: DISSECTING THE WRAPPING ISSUE \\ Reply to the Editor:}

In their letter to the Editor, Lin and colleagues ${ }^{1}$ correctly argue that the use of the Cabrol fistula in all aortic reconstructions not only is not necessary but may prove complicated and dangerous. Extended aortic reconstructions involving the arch, especially if using trifurcated or quadrifurcated grafts would be especially difficult to reconstruct a fistula. Closure of the spaces between the individual arms of the grafts and the superior thoracic inlet spaces would be a complex and time-consuming effort, and, as is pointedly discussed in the letter, may require extensive dissection and mobilization, if not transection, of the innominate vein to facilitate construction. Although these manipulations might be problematic, the majority of significant, difficult-to-reach bleeding typically arises from the periroot segment of the aorta, especially the left coronary button. As is again correctly discussed, the need for reflecting and repositioning various remnants of residual aorta would put excessive strain on delicate tissues already traumatized by suture, potentially creating a catastrophic result. Further, current technique and comfort with aortic reconstructive measures have resulted in, even at low-volume centers, acceptable results that are not plagued with significant bleeding issues, again obviating the need for extensive shunting procedures.

Fundamentally, as iterated, protecting the patient's life is of foremost concern in all procedures, and the use of the Cabrol fistula has undoubtedly salvaged many aortic repairs. The need for the application of this technique to all patients, however, Lin and colleagues ${ }^{1}$ correctly argue, may be overwrought if meticulous technique and careful attention to detail are applied throughout the reconstructive process.

Jan D. Galla, $M D, P h D$

Englewood Cardiac Surgery Associates Englewood Hospital Medical Center Englewood, NJ 